\title{
Classical Nuclear Motion in Quantum Transport
}

\author{
Claudio Verdozzi* Gianluca Stefanucci, and Carl-Olof Almbladh \\ Solid State Theory, Lund University, Sölvegatan 14 A, 22362 Lund, Sweden
}

(Dated: May 10, 2018)

\begin{abstract}
An $a b$ initio quantum-classical mixed scheme for the time evolution of electrode-device-electrode systems is introduced to study nuclear dynamics in quantum transport. Two model systems are discussed to illustrate the method. Our results provide the first example of current-induced molecular desorption as obtained from a full time-dependent approach and suggest the use of ac biases as a way to tailor electromigration. They also show the importance of non-adiabatic effects for ultrafast phenomena in nanodevices.

PACS numbers: 72.10.Bg, 73.63.-b, 63.20.Ry, 63.20.Kr
\end{abstract}

The study of electron-nuclei interaction (ENI) in solids has a long and important history, dating back to the early days of quantum mechanics. Since then, the ENI has become a pillar concept of our understanding in condensed matter [1]. Increase in computer power and the introduction of ab-initio molecular dynamics [2] have made possible quantitative studies of ENI in many materials.

Recent advances in nanotechnology pose new questions about the ENI in out-of-equilibrium open systems at the nanoscale [3]. Among the techniques used to study the ENI in this context, quantum transport experiments stand out as a special case, since charge conduction is at the same time a way to characterize the nanodevice and a property to be exploited in its operating regime. A case in point is molecular junctions, where electron injection can stimulate local vibrations [4, 5] and possibly electromigration [6]. More generally, describing ENI in time-dependent (TD) quantum transport is expected to become of great technological interest, since future nanodevices will operate under the influence of ever faster time varying external fields. Accordingly, those regarded at present as marginal transient effects will soon become center stage features. Assessing and engineering the ENI is then a key ingredient to increase the device efficiency [7, 8]. To date, most theoretical studies have addressed the ENI in steady-state phenomena $9,10,11,12,13,14,15$ and often perturbatively in the nuclear displacements 13, 14, 15. Going beyond the harmonic approximation and including the ENI in a firstprinciples, TD framework is a difficult theoretical task which has received so far scarce attention [16, 17, 18, 19].

In this work we propose a first principle approach to TD quantum transport which treats the nuclear motion in the Ehrenfest dynamics (ED), and the electrons within Time-Dependent Density Functional Theory (TDDFT) 20]. The ED has been extensively used in several contexts; in quantum transport, it was considered in [18]. The ED correctly displays many important features of ENI, but gives an incomplete account of the Joule heating of the nuclei by the electrons [19]. However, such effect is not the aim of this first work. Here we use ED which, while treating ENI at the mean field level, includes, as opposed to the Born Oppenheimer Approximation, electronic transitions. Our approach to transport permits the description of transient effects: it can be used to describe history dependent currents, hysteresis phenomena, etc. Another main advantage is that it can perform the TDDFT-ED of devices connected to infinitely long leads.

We illustrate the approach with two model devices, where the electrons interact only via the ENI: a Holstein wire (D1) and a diatomic molecule (D2). We choose these two rather different systems to show the versatility of our method in discussing transient phenomena and overcoming the limitations of adiabatic treatments. More in detail, our results show that: (i) For weak electron-phonon (e-ph) couplings, D1 exhibits an almost periodic nuclear displacement (with period $=1 /$ density), reminiscent of a Peierls distortion. On applying a dc bias, the nuclei oscillate (with decreasing amplitude) and the period changes to accommodate the current flow. On increasing the $e$ ph coupling, D1 changes from conducting to insulating. (ii) D2 is deformed by a small, suddenly switched on dc bias. Above a critical value of the bias, the molecule dissociates. This is the single most important result of this work. To our knowledge, it provides the first example of current-induced molecular desorption as emerging from the full TD dynamics of a nanodevice. (iii) The desorption cannot be described in any adiabatic formalism, since it is due to electronic excitations induced by the nuclear motion. (iv) The desorption can be tuned by the intensity and frequency of an ac bias, suggesting a way to control electromigration in molecular devices.

The Method. Following Refs. [21, 22, 23], in the initial ground state the central region $(\mathrm{C})$ is in contact to seminfinite left $(\mathrm{L})$ and right $(\mathrm{R})$ leads. With ENI, the Hamiltonian for $\mathrm{C}$ reads

$$
\hat{H}_{\mathrm{C}}[\mathbf{x}]=\sum_{i, j=1}^{M} V_{i j}(\mathbf{x}) c_{i}^{\dagger} c_{j},
$$

where $M$ is the number of one-electron states of $\mathrm{C}$ and $\mathbf{x}=\left(x_{1}, \ldots, x_{N}\right)$ are the nuclear coordinates. Outside $\mathrm{C}$, the nuclei are clamped. In the Kohn-Sham (KS) scheme 
of TDDFT [20], $V_{i j}$ would be the $(i, j)$ matrix element of the KS Hamiltonian. The nuclear classical Hamiltonian is $H_{\mathrm{cl}}=\sum_{k=1}^{N} p_{k}^{2} /\left(2 m_{k}\right)+U_{\mathrm{cl}}(\mathbf{x})$. The dynamics of the system is governed by

$$
\begin{aligned}
i \frac{\mathrm{d}}{\mathrm{d} t}|\Psi\rangle & =\hat{H}_{\mathrm{el}}|\Psi\rangle, \\
m_{k} \frac{\mathrm{d}^{2} x_{k}}{\mathrm{~d} t^{2}} & =-\frac{\partial}{\partial x_{k}}\left(U_{\mathrm{cl}}+\left\langle\Psi\left|\hat{H}_{\mathrm{C}}\right| \Psi\right\rangle\right),
\end{aligned}
$$

where $|\Psi(t)\rangle$ is the many-electron state at time $t$, and $\hat{H}_{\mathrm{el}}[\mathbf{x}]$ is the electron Hamiltonian of the contacted system $\mathrm{L}+\mathrm{C}+\mathrm{R}$. Given a configuration $\mathbf{x}, \hat{H}_{\mathrm{el}}[\mathbf{x}]$ is a freeparticle Hamiltonian. The many-electron ground state $\left|\Psi_{g}[\mathbf{x}]\right\rangle$ consists of bound, resonant, fully reflected waves, plus left and right going scattering states. The parametric dependence of $\hat{H}_{\mathrm{el}}$ on $\mathbf{x}$ renders every eigenstate a function of $\mathbf{x}$. The ground state value $\mathbf{x}=\mathbf{x}_{g}$ is computed using a damped ground-state dynamics: starting from an initial $\mathbf{x}_{0}$ the coordinates are evolved according to $m_{k} \ddot{x}_{k}=-\gamma \dot{x}_{k}-\partial\left(U_{\mathrm{cl}}+\left\langle\Psi_{g}\left|\hat{H}_{\mathrm{C}}\right| \Psi_{g}\right\rangle\right) / \partial x_{k}$, with $\gamma$ the friction coefficient.

Having $\mathbf{x}_{g}$ and the corresponding $\{\psi\}$ one-electron orbitals, we apply an external bias and evolve the system. Assuming metallic electrodes and instantaneous screening, the size of $\mathrm{C}$ is chosen so that the potential drop at any time occurs entirely in C. Thus, the TD part of the electrode Hamiltonian is a spatially uniform shift $U_{\eta}(t), \eta=\mathrm{L}, \mathrm{R}$. We use a novel mixed quantum-classical evolution algorithm, which combines a recently proposed generalization of the Crank-Nicholson method 23] for the $\{\psi\}$ with a Verlet-like integrator for the $\mathbf{x}$. Schematically, in terms of the discretized time $t_{m}=2 m \delta$,

$$
\left\{\begin{array}{l}
\left\{\psi^{(m+1)}\right\}=\left\{\boldsymbol{S}\left[t_{m}, \mathbf{x}^{(m)}\right] \psi^{(m)}\right\} \\
\left\{\begin{array}{l}
p_{k}^{(m+1)}=p_{k}^{(m)}+2 \delta F_{k}\left[\mathbf{x}^{(m)},\left\{\psi^{(m+1)}\right\}\right] \\
x_{k}^{(m+2)}=x_{k}^{(m)}+4 \delta p_{k}^{(m+1)} / m_{k} \\
p_{k}^{(m+2)}=p_{k}^{(m+1)}+2 \delta F_{k}\left[\mathbf{x}^{(m+2)},\left\{\psi^{(m+1)}\right\}\right]
\end{array}\right. \\
\left\{\psi^{(m+2)}\right\}=\left\{\boldsymbol{S}\left[t_{m+1}, \mathbf{x}^{(m)}\right] \psi^{(m+1)}\right\}
\end{array}\right.
$$

with $\psi^{(m)}=\psi\left(t_{m}\right), \mathbf{x}^{(m)}=\mathbf{x}\left(t_{m}\right)$ and $\mathbf{p}^{(m)}=\mathbf{p}\left(t_{m}\right)$. The unitary matrix $\boldsymbol{S}$ depends on time through $\mathbf{x}(t)$ and the TD bias $U_{\eta}(t), \eta=\mathrm{L}, \mathrm{R}$. Full details of the electronic evolution can be found in Ref. 23]. The force $F[\mathbf{x},\{\psi\}]$ is given by the r.h.s. of Eq. (3).

To illustrate the method, we describe electrodes L and $\mathrm{R}$ in terms of one-dimensional tight-binding (TB) Hamiltonians with a hopping $V$ between nearest neighbor sites (TB parametrization of $\mathrm{Au}$ wires suggest $|V|$ in the range $0.3 \div 1.0 \mathrm{eV}$ ). Left and right going scattering states have energy $\varepsilon$ within the band $(-2|V|, 2|V|)$ and can be obtained by solving the Schrödinger equation in $\mathrm{C}$ with appropriate boundary conditions. Bound state eigenenergies $\varepsilon_{b}<-2|V|$ satisfy Det $\left[\varepsilon_{b} \mathbf{1}-\boldsymbol{H}_{\mathrm{C}}-\boldsymbol{\Sigma}\left(\varepsilon_{b}\right)\right]=0$, and the associated wavefunction is given in $\mathrm{C}$ by the kernel of $\left[\varepsilon_{b} \mathbf{1}-\boldsymbol{H}_{\mathrm{C}}-\boldsymbol{\Sigma}\left(\varepsilon_{b}\right)\right]\left(\boldsymbol{H}_{\mathrm{C}}\right.$ is the projection of $\hat{H}_{\mathrm{C}}$ onto the one-electron Hilbert space, $\boldsymbol{\Sigma}(\omega)$ is the embedding self-energy). The topology of region $\mathrm{C}$ might also lead to states rigorously confined in $\mathrm{C}$ (see below). These are resonant eigenstates of the uncontacted $\boldsymbol{H}_{\mathrm{C}}$ with zero amplitude at the interface with the two electrodes.

Model device D1. The semiclassical Holstein model is a valuable tool to gain insight into $e$-ph interactions [24, 25]. Here, we investigate TD transport through a Holstein wire described by

$$
\hat{H}_{\mathrm{C}}=V \sum_{i=-M}^{M-1}\left(c_{i}^{\dagger} c_{i+1}+\text { h.c. }\right)-g \sum_{i=-M}^{M} x_{i} \hat{n}_{i},
$$

where $\hat{n}_{i}=c_{i}^{\dagger} c_{i}$ is the local density operator and $x_{i}$ are the phonon coordinates. The nuclear classical potential
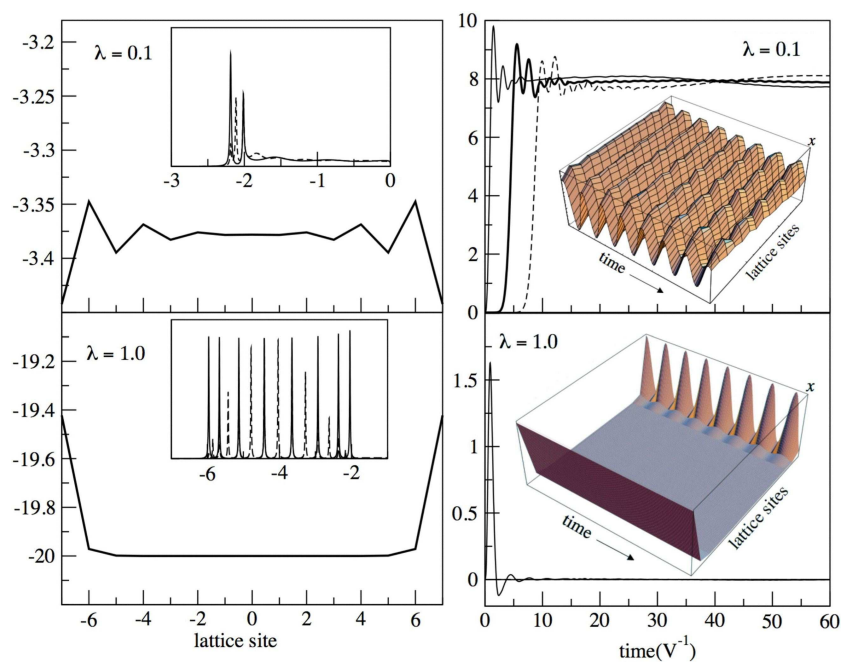

FIG. 1: Left panel: Ground state displacement $x_{i}, i=$ $-7, \ldots, 7$, at weak $(\lambda=0.1)$ and strong $(\lambda=1.0)$ coupling. The inset shows the LDOS (energy in units of $|V|$ ) on site -6 (dashed line) and site 0 (solid line). Right panel: current $I(t)$ (in units of $10^{-2}|V|$ ) at weak and strong coupling along the bond $(-7,-6)$ (solid thin), $(0,1)$ (solid thick) and $(6,7)$ (dashed) after the sudden switching on of a bias $U_{\mathrm{L}}=0.5|V|$ in electrode $\mathrm{L}$. The insets show a $3 \mathrm{D}$ plot of $\mathbf{x}(t)$ between $t=0$ and $t=480|V|^{-1}$ (in the top panel the range of $\mathrm{x}$ is between -3.2 and -4 while in the bottom panel is between -19.4 and -20 ).

is $U_{\mathrm{cl}}(\mathbf{x})=\frac{1}{2} \sum_{i=-M}^{M} m_{i} \omega_{0}^{2} x_{i}^{2}$. The strength of the $e$-ph interaction is determined by the dimensionless parameter $\lambda=g^{2} /\left(2 V \omega_{0}\right)$. In the original derivation by Holstein, $x_{i}$ corresponds to an internal coordinate (bond-length) at the $i$-th site. We study D1 at half-filling $\left(\varepsilon_{\mathrm{F}}=0\right)$, in the adiabatic regime $\left(\alpha=\omega_{0} / V=0.1\right)$ at weak $(\lambda=0.1)$ and strong $(\lambda=1)$ coupling. A damped ground-state dynamics, as described above, was used to get the converged ground-state $x_{i}$ (Fig. 1 left) for a region $\mathrm{C}$ with $M=7$. The energy spectrum between $-2|V|$ and $\varepsilon_{\mathrm{F}}$ was discretized and good convergence was achieved with 
$N_{k}=500$ meshpoints. For $\lambda=0.1$, a Peierls-like distortion is seen: an even-odd behavior of $x_{i}$ is manifest (in general, $P=1 / n$, and here $n=0.5$ ), but exact periodicity is prevented by the finite size of $\mathrm{C}$. The inset shows the local density of states (LDOS) close to the interface and in the center, respectively. At $\varepsilon<-2|V|$ we observe three peaks due to three bound states. The picture changes dramatically at $\lambda=1$ : The number of bound states equals the dimension of $\mathrm{C}$ and, almost uniformly, $x_{i} \simeq-20$. Only the $x_{i}$ close to the interfaces are slightly above this value.

In Fig. 11 right, we plot the current $I(t)$ in three different points of $\mathrm{C}$ after suddenly switching on a bias $U_{\mathrm{L}}=0.5|V|$ in the left electrode. All calculations use a time step $\delta=0.01|V|^{-1}$. For both $\lambda=0.1,1.0$, the current in the (short) transient is similar to the case with the $x_{i}$ clamped (not shown), since electrons are much faster than nuclei. At $\lambda=0.1$ the steady current shows superimposed oscillations of frequency $\omega_{0}$. Instead, for $\lambda=1$, all the $2 M+1=15$ bound states in $\mathrm{C}$ are occupied (including the Hartree potential would have led to a significant reduction of this excess density in $\mathrm{C}$ ), no current fluctuations occur in the center and at the right interface and only a very short transient is observed at the left interface. The insets display the TD Peierls distortion. For $\lambda=0.1$ all the $x$ 's oscillate with an exponentially decreasing amplitude (within our simulation time $t=480|V|^{-1}$ ). The overall shape of the $x_{i}$ changes to accommodate the net electron flow. For $\lambda=1$ no current flows and only the $x_{i}$ close to the left interface oscillate. The decay of the amplitudes of the $x_{i}$ is partially due to the inefficiency of ED in transferring energy from electrons to nuclei. We also considered the sudden removal of a bound electron, as obtainable for example by optical means: in this case D1 provides a strong transient oscillating response (not shown). On speculative grounds, such behavior could be used for ENI based photosensors.

Model device D2. We consider a central region $\mathrm{C}$ with the simplest non-trivial topology, a four-atom ring. This is our model molecular device D2, with nuclear positions $\mathbf{r}_{i} \equiv\left(x_{i}, y_{i}\right), i=1, . ., 4$ (Fig. 2] top-left). In D2, only nuclei 2 and $3(\mathrm{~N} 2,3)$ are let to move in the $x y$ plane. The origin of the $x y$ plane is the midpoint of nuclei 1 and 4. For the hopping parameter we choose the form [26] $V_{i \neq j}=V_{c} \frac{e^{-r_{i j}}}{r_{i j}}, V_{i=j}=0$, where $r_{i j}=\left|\mathbf{r}_{i}-\mathbf{r}_{j}\right|$. The purely repulsive classical term is given by $U_{\mathrm{cl}}=\frac{1}{2} \sum_{i \neq j} A / r_{i j}^{4}$. We tune the parameters $V_{c}, A$ and $r_{14}$ to ensure a reasonable domain of structural stability for D2. For $V_{c}=4 V$, $A=0.75|V|$ and $r_{14}=2$, D2 is stable against deformations up to $\sim 10 \%$ of the equilibrium distances. Also, we get the ground state positions $\mathbf{r}_{2}=(0,0.8313)$ and $\mathbf{r}_{3}=-\mathbf{r}_{2}$ (the $2-3$ symmetry remains true in the presence of the bias). The LDOS has one peak below $-2|V|$, i.e. one bound state. There is also a resonant state $|\psi\rangle=\frac{1}{\sqrt{2}}(|2\rangle-|3\rangle)$ with energy $\left|V_{23}\right|>0$ inside the band.

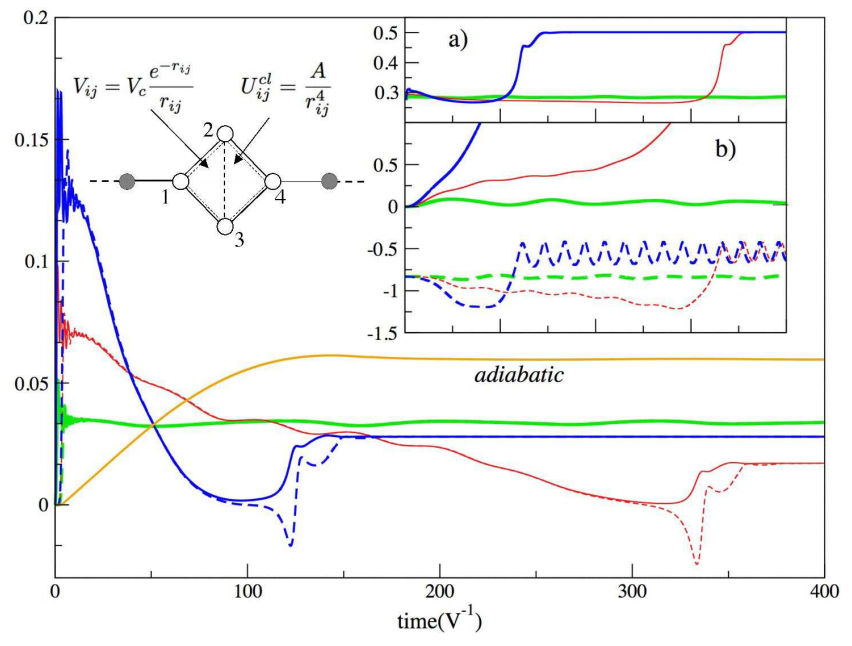

FIG. 2: $I_{\mathrm{L}, \mathrm{R}}(t)$ (in units of $|V|$ ) at the left (solid line) and right (dashed line) interface for a sudden switching $U_{\mathrm{L}}=0.25|V|$ (green[light grey]), $0.5|V|$ (red [thin dark grey]) and 1.0|V| (blue [thick black]). Inset a): Time dependent density $n_{3}$ of nucleus 3 (by symmetry $n_{2}=n_{3}$ at any time). Inset b): $x_{3}(t)$ (solid line) and $y_{3}(t)$ (dashed line) of nucleus 3 (by symmetry $x_{2}=x_{3}$ and $y_{2}=-y_{3}$ at any time). Time scale and color coding in insets a) and b) is the same as in the main figure. A schematic of the device D2 and the adiabatic result (labeled curve) are also shown.

The system is taken at half filling, and we switch on a bias $U_{\mathrm{L}}$ at $t=0$. As for D1, $\delta=0.01|V|^{-1}, N_{k}=500$; for the masses of $\mathrm{N} 2,3$ we choose $100|V|^{-1}$.

In Fig. 2] we plot the TD currents $I_{\mathrm{L}}$ and $I_{\mathrm{R}}$ at the left and right interfaces, respectively. At small bias $U_{\mathrm{L}}=0.25|V|, I_{\mathrm{L}, \mathrm{R}}$ rapidly increase and after a short transient (with the nuclei essentially still) start to oscillate around a steady value. Inset b) shows the corresponding nuclear dynamics. The equilibrium rhombic geometry changes and the molecule gets deformed in the biased system, with N2,3 having damped oscillations around two new positions (for the damping and $\mathrm{ED}$, considerations similar to the case of D1 apply). We also notice from inset a) that the charge density of N2,3 slightly increases.

Highly interesting is the strong bias case $U_{\mathrm{L}}=1.0|\mathrm{~V}|$. After a very short transient, $I_{\mathrm{L}, \mathrm{R}}$ sharply decrease (rather than oscillating around a steady value) and become zero at $t_{0} \simeq 100|V|^{-1}$. After $t_{0}, I_{\mathrm{L}, \mathrm{R}}$ separate: $I_{\mathrm{L}}$ increases while $I_{\mathrm{R}}$ decreases, reaches a negative minimum and then increases to eventually re-join $I_{\mathrm{L}}$ at $t_{1} \sim 160|V|^{-1}$. For $t>t_{1}, I_{\mathrm{L}} \simeq I_{\mathrm{R}}$ and their value equal the steady current (calculated from the Landauer formula) of the chain without $\mathrm{N} 2,3$. The behavior of $I_{\mathrm{L}, \mathrm{R}}$ can be understood looking at the nuclear dynamics (inset b): the force exerted by the electron flow is strong enough for atomic migration to occur. N2,3 are pushed to the right by the current, overcome the confining potential and get dissociated from region C. Thereafter, they form a diatomic 


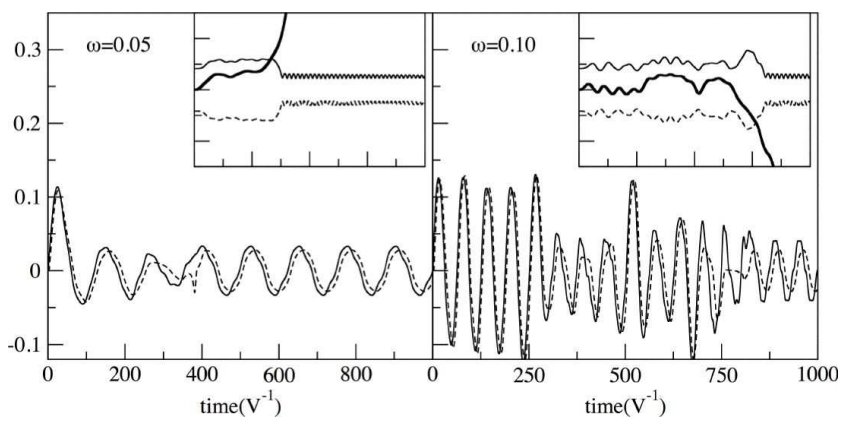

FIG. 3: Left panel: $I_{\mathrm{L}, \mathrm{R}}(t)$ (in units of $|V|$ ) at the left (solid line) and right (dashed line) interface for $U_{L}=1.0|V|, \omega=$ $0.05|V|$. In the inset, $x_{2}(t)=x_{3}(t)$ (thick solid) and $y_{2}(t)$ (thin solid), $y_{3}(t)$ (dashed). The time scale is the same as in the main panel. Right panel: same as left, but with $\omega=$ $0.10|V|$. Left and right insets have the same vertical scale .

molecule vibrating along $y$ (see inset b) and traveling along $x$ at uniform speed. The pronounced minimum of $I_{\mathrm{R}}$ shortly after $t_{0}$ is due to a sudden charge transfer from electrode R (via atom 4) to the diatomic molecule when $\mathrm{N} 2,3$ pass above nucleus 4 . This is confirmed in inset a) where the density suddenly increases in correspondence of the minimum in $I_{\mathrm{R}}$. We also note that the total density of the dissociated molecule is about 1 (exact charge quantization would occur in the adiabatic approximation only). Here, including the Hartree potential would not lead to qualitative changes of the dissociation process. A more realistic model should also include the possibility for the molecule to chemisorb onto the electrode, to fragment etc., but this is beyond the scope of the present Letter.

To address the dissociation mechanism, we study two different $U_{\mathrm{L}}(t)$, with the same asymptotic value $0.5|V|$. For a sudden switching, one observes a behavior delayed but similar to the $1.0|V|$ case above. Instead, for an adiabatic switching 27] the dimer does not dissociate ( in Fig. 2 the current is displayed in orange and labeled "adiabatic"). We conclude that electronic excitations induced by the nuclear motion play a crucial role in the electromigration, a role that can not be accounted for by any adiabatic formalism (without transients the nuclei experience no force). However, we observe that ED might overestimate the value of the critical bias for which dissociation occurs.

We next examined the response of $\mathrm{D} 2$ when subject to a high amplitude ac bias $U_{\mathrm{L}}(t)=U_{\mathrm{L}} \sin (\omega t)$, with $U_{\mathrm{L}}=1.0|V|$. At low $\omega$ (Fig. [3 left), the system qualitatively behaves as in the dc case, as clear from both current (main left) and coordinates (inset left) panels. The nuclei overtake the barrier before the change in $U_{\mathrm{L}}(t)$ produces a force able to "recall" them back. After the desorption, $I_{\mathrm{L}, \mathrm{R}}$ oscillate as they would for a linear chain without N2,3. At larger $\omega$ (Fig. 3 right) the dissociation is delayed, since atoms 2 and 3 are recalled back a few times before leaving region C. Eventually, for high enough frequency $(>0.3|V|$, not shown) atoms 2 and 3 oscillate without leaving $\mathrm{C}$ (within our simulation time). These results point to a possible use of ac biases as a way to tailor molecular desorption in nanodevices.

In conclusion, we presented a mixed quantumclassical scheme to describe electron-nuclei interactions in quantum transport. The scheme is straightforwardly amenable to an ab-initio implementation. Our results show the necessity to go beyond adiabatic schemes and to use a full time-dependent, nuclear-dynamics approach for ultrafast transient phenomena which are expected to become important in future generation nanodevices. We acknowledge useful discussions with G. J. Ackland, P. Bokes and E. N. Economou. This work was supported by the European Community 6th framework Network of Excellence NANOQUANTA (NMP4-CT-2004-500198).

* Electronic address: cv@teorfys.lu.se

[1] J. M. Ziman, Electron and Phonons, Oxford U. P. (1960).

[2] R. Car, and M. Parrinello, Phys. Rev. Lett. 55, 2471 (1985).

[3] Electron-Phonon Interactions in Low-Dimensional Structures, L. Challis ed., Oxford U. P., Oxford (2003).

[4] M. A. Reed, C. Zhou, C. J. Muller, T. P. Burgin, and J. M. Tour, Science 278, 252 (1997).

[5] H. Park, J. Park, A. K. L. Lim, E. H. Anderson, A. P. Alivisatos and P. L. McEuen, Nature 407, 57 (2000).

[6] H. Yasuda and A. Sakai, Phys. Rev. B 56, 1069 (1997).

[7] P. Hyldgaard, Low Temp. Phys. 27, 585 (2001).

[8] A. A. Balandin, J. Nanosci. Nanotechnol. 5, 1015 (2005).

[9] E. G. Emberly and G. Kirczenow, Phys. Rev. B 64, 125318 (2001).

[10] T. N. Todorov, J. Hoekstra, and A. P. Sutton, Phys. Rev. Lett. 86, 3606 (2001).

[11] M. Brandbyge, K. Stokbro, J. Taylor, J. L. Mozos, and P. Ordejon, Phys. Rev. B 67, 193104 (2003).

[12] M. Di Ventra, S. T. Pantedelis and N. D. Lang, Phys. Rev. Lett. 88, 046801 (2002).

[13] M. Paulsson, T. Frederiksen, and M. Brandbyge, Phys. Rev. B 72, 201101(R), 2005.

[14] T. Frederiksen, M. Brandbyge, N. Lorente and A.-P. Jauho, Phys. Rev. Lett. 93, 256601 (2004).

[15] K. Burke, R. Car and R. Gebauer, Phys. Rev. Lett. 94, 146803 (2005).

[16] Z. G. Yu, D. L. Smith, A. Saxena and A. R. Bishop, Phys. Rev. B 59, 16001 (1999).

[17] R. Lu and Z. R. Liu, J. Phys. Condens. Matter 17, 5859 (2005).

[18] A. P. Horsfield, D. R. Bowler, A. J. Fisher, J. Phys. Condens. Matter 16, L65 (2004).

[19] A. P. Horsfield, D. R. Bowler, A. J. Fisher, T. N. Todorov, and C. G. Sanchez, J. Phys. Condens. Matter 16, 8251 (2004).

[20] E. Runge and E. K. U. Gross, Phys. Rev. Lett. 52, 997 (1984).

[21] M. Cini, Phys. Rev. B 22, 5887 (1980).

[22] G. Stefanucci and C.-O. Almbladh, Phys. Rev. B 69, 
195318 (2004).

[23] S. Kurth, G. Stefanucci, C.-O. Almbladh, A. Rubio and E. K. U. Gross, Phys. Rev. B 72, 035308 (2005).

[24] S. Kumar and P. Majumdar, Phys. Rev. Lett. 94, 136601 (2005).

[25] G. Kopidakis, C. M. Sokoulis and E. N. Economou, Phys. Rev. B 51, 15038 (1995).
[26] L. Goodwin, A. J. Skinner and D. G. Pettifor, Europhys. Lett. 9,701 (1989).

[27] We take $U_{\mathrm{L}}(t)=0.5|V| \sin \left(\pi t / 2 t_{0}\right), t \leq t_{0}=150|V|^{-1}$. To smoothen the switching as much as possible, we damp the nuclear motion using a friction $\gamma=0.07$ up to $t<200|V|^{-1}$ and let the system evolve without friction afterwards. 